\title{
Some sufficient conditions for solvability of the Dirichlet problem for the complex Monge-Ampère operator
}

\author{
by SŁawomir KoŁodziej (Kraków)
}

\begin{abstract}
We find a bounded solution of the non-homogeneous Monge-Ampère equation under very weak assumptions on its right hand side.
\end{abstract}

Introduction. In this paper we are interested in solving, under possibly weak assumptions on the measure $d \mu$, the following Dirichlet problem for the complex Monge-Ampère equation in a given strictly pseudoconvex domain $\Omega \subset \mathbb{C}^{n}:$

$$
\begin{aligned}
& u \in \mathrm{PSH} \cap L^{\infty}(\Omega), \\
& \left(d d^{c} u\right)^{n}=d \mu, \\
& \lim _{z^{\prime} \rightarrow z} u\left(z^{\prime}\right)=\phi(z), \quad z \in \partial \Omega, \phi \in C(\partial \Omega),
\end{aligned}
$$

where $d=\partial+\bar{\partial}, d^{c}=i(\bar{\partial}-\partial)$ and so $d d^{c}=2 \pi i \partial \bar{\partial}$. It has been shown by E. Bedford and B. A. Taylor [BT1] that the wedge product $\left(d d^{c} u\right)^{n}=$ $d d^{c} u \wedge \ldots \wedge d d^{c} u$ is well defined for plurisubharmonic (psh), locally bounded functions $u$, and that $(*)$ is solvable for measures having continuous densities with respect to the Lebesgue measure (here denoted by $d \lambda$ ). The equation has attracted attention of a number of authors; we refer to [B] for a more detailed account. In particular, it is known that continuous solutions exist if $d \mu=f d \lambda$, where $f \in L^{2}(\Omega, d \lambda)$ (U. Cegrell-L. Persson [CP]), but for $f \in$ $L^{1}(\Omega, d \lambda)$ this is not necessarily true [CS]. In Theorem 3 below we show that if $f \in L^{p}(\Omega, d \lambda), p>1$, then there exists a continuous solution of $(*)$. This is the answer to the question posed in $[\mathrm{CS}]$ and $[\mathrm{P}]$ (see also $[\mathrm{B}],[\mathrm{BL}]$ ). For the case of rotation invariant measures in a ball a solution was given in $[\mathrm{P}]$. The result can be extended from $L^{p}, p>1$, to some Orlicz spaces as shown

\footnotetext{
1991 Mathematics Subject Classification: Primary 32F07; Secondary 32F05.

Key words and phrases: plurisubharmonic function, complex Monge-Ampère operator. Research partially supported by KBN Grant 2 P03A 05809.
} 
in Theorem 4. To prove it we use an a priori estimate for the $\|u\|_{L^{\infty}}$ norm of a solution of $(*)$ if $d \mu$ satisfies a certain integral condition (Theorem 1 ). E. Bedford [B] conjectured that some such estimate is possible. It is shown that the integral condition cannot be substantially weakened. Combining Theorem 1 with the results of $[\mathrm{KO}]$ we solve the Dirichlet problem $(*)$ for a large family of measures $d \mu$.

I am indebted to my colleagues Z. Błocki and G. Lewicki for valuable suggestions and U. Cegrell (Umeå University) for pointing out an error in a previous version of the paper. The referee's remarks simplified the proof of Theorem 1.

Preliminaries. Here we present some notions and results which are used in the paper. The background material can be found in [B], [K], [S]. $\Omega$ will denote throughout a strictly pseudoconvex domain in $\mathbb{C}^{n}$. For a compact subset $K \subset \Omega$ we define the relative extremal function and the relative capacity [BT2] (see also [B], [K]) by the formulas

$$
\begin{gathered}
u_{K}(z)=\sup \left\{u(z): u \in \operatorname{PSH} \cap L^{\infty}, u<0 \text { in } \Omega, u \leq-1 \text { on } K\right\}, \\
\operatorname{cap}(K, \Omega)=\sup \left\{\int_{K}\left(d d^{c} u\right)^{n}: u \in \operatorname{PSH}(\Omega),-1 \leq u<0\right\} .
\end{gathered}
$$

By [BT2],

$$
\operatorname{cap}(K, \Omega)=\int_{K}\left(d d^{c} u_{K}^{*}\right)^{n}=\int_{\Omega}\left(d d^{c} u_{K}^{*}\right)^{n},
$$

where $u_{K}^{*}:=\varlimsup_{z^{\prime} \rightarrow z} u_{K}(z)$. If $u_{K}^{*}=u_{K}$ we say that $K$ is regular. For an open subset $U \subset \Omega$ the relative capacity is defined by

$$
\operatorname{cap}(U, \Omega)=\sup \{\operatorname{cap}(K, \Omega): K \subset U, K \text { compact }\} .
$$

Another extremal function (of logarithmic growth) and an associated capacity were introduced by J. Siciak (see $[\mathrm{S}],[\mathrm{AT}],[\mathrm{B}],[\mathrm{K}]$ ):

$$
\begin{aligned}
& L_{K}(z)=\sup \left\{u(z): u \in \operatorname{PSH}\left(\mathbb{C}^{n}\right),\right. \\
&u(z)<\log (1+|z|)+O(1), u \leq 0 \text { on } K\}, \\
& T_{R}(K):=\exp \left(-\sup \left\{L_{K}^{*}(z):|z| \leq R\right\}\right)
\end{aligned}
$$

for a compact set $K \subset \mathbb{C}^{n}$ and a given $R>0$. We extend the definition of $T_{R}$ to open sets in the same way as the definition of cap above.

Important inequalities between cap and $T$ were proved by $\mathrm{H}$. Alexander and B. A. Taylor [AT]. If $B:=B(0, R)$ and $K \subset B(0, r), r<R$, is compact, then

$$
\exp \left(-A(r)(\operatorname{cap}(K, B))^{-1}\right) \leq T_{R}(K) \leq \exp \left(-2 \pi(\operatorname{cap}(K, B))^{-1 / n}\right) .
$$

The main tool in pluripotential theory is the following Comparison Principle of Bedford and Taylor [BT2]: 
Comparison PRINCIPLe. If $u, v \in \operatorname{PSH} \cap L^{\infty}(\Omega)$ and $\liminf _{z \rightarrow \partial \Omega}(u(z)$ $-v(z)) \geq 0$, then

$$
\int_{\{u<v\}}\left(d d^{c} v\right)^{n} \leq \int_{\{u<v\}}\left(d d^{c} u\right)^{n} .
$$

Due to the same authors and presented here in a simplified version, sufficient for our applications, is

Convergence Theorem [BT2]. If $u_{j} \in \mathrm{PSH} \cap L^{\infty}(\Omega), j=1,2, \ldots$, and $u_{j} \uparrow u$ a.e. in $\Omega$ or $u_{j} \downarrow u$ with $u \in \operatorname{PSH} \cap L_{\mathrm{loc}}^{\infty}(\Omega)$ then

$$
\left(d d^{c} u_{j}\right)^{n} \rightarrow\left(d d^{c} u\right)^{n}
$$

in the sense of currents.

An a priori estimate. We begin with proving an a priori estimate for the $L^{\infty}$ norm of a solution to the Dirichlet problem $(*)$ when $d \mu$ is assumed to satisfy a certain integral condition.

TheORem 1. Let $\Omega$ be a strictly pseudoconvex domain in $\mathbb{C}^{n}$ and let $\mu$ be a Borel measure in $\Omega$ such that $\int_{\Omega} d \mu \leq 1$. Consider an increasing function $h: \mathbb{R} \rightarrow(1, \infty)$ satisfying

$$
\int_{1}^{\infty}\left(y h^{1 / n}(y)\right)^{-1} d y<\infty
$$

If $\mu$ satisfies the integral condition

$$
\int_{\Omega}|v|^{n} h(|v|) d \mu \leq A
$$

whenever

$$
v \in \operatorname{PSH}(\Omega) \cap C(\bar{\Omega}), \quad v=0 \text { on } \partial \Omega, \quad \int_{\Omega}\left(d d^{c} v\right)^{n} \leq 1,
$$

then the norm $\|u\|_{L^{\infty}}$ of a solution of the Dirichlet problem $(*)$ is bounded by a constant $B=B(h, A)$ which does not depend on $\mu$.

Proof. It is no restriction to assume that $\phi=0$ in $(*)$ : the general case will follow by the Comparison Principle [BT2]. Let $u$ be a solution of $(*)$. For $s<0$ denote by $U_{s}$ the open set $\{u<s\}$ and put

$$
a(s):=\operatorname{cap}\left(U_{s}, \Omega\right)=\operatorname{cap}\left(U_{s}\right), \quad b(s):=\mu\left(U_{s}\right) .
$$

Our proof rests on the following two propositions.

Proposition 1. $b(s) \leq A a(s) h^{-1}\left([a(s)]^{-1 / n}\right)$.

Proposition 2. $t^{n} a(s) \leq b(s+t)$ if $t>0$ and $s+t<0$. 
Proof of Proposition 1. Consider $v=(r a(s))^{-1 / n} u_{K}$, where $K \subset U_{s}$ is a compact regular set with $\operatorname{cap}(K)=r a(s)(r<1)$. Then $\int\left(d d^{c} v\right)^{n}=1$ and so the integral condition $(* *)$ applies, giving

$$
A \geq \int_{\Omega}|v|^{n} h(|v|) d \mu \geq \int_{K}|v|^{n} h(|v|) d \mu=(r a(s))^{-1} h\left([r a(s)]^{-1 / n}\right) \mu(K),
$$

which is just the desired estimate as $r \rightarrow 1$ (and so $\mu(K) \rightarrow b(s)$ ).

Proof of Proposition 2. We apply the Comparison Principle [BT2] to the pair of functions $u_{K}$ and $v:=(r t)^{-1}(u-s-t)$, where $K, r$ are defined as above. Note that $K \subset\left\{v<u_{K}\right\} \subset U_{s+t}$. Hence

$$
\begin{aligned}
r a(s) & =\int_{\left\{v<u_{K}\right\}}\left(d d^{c} u_{K}\right)^{n} \leq(r t)^{-n} \int_{\left\{v<u_{K}\right\}}\left(d d^{c} u\right)^{n} \\
& \leq(r t)^{-n} \mu\left(U_{s+t}\right)=(r t)^{-n} b(s+t) .
\end{aligned}
$$

The proposition follows if we let $r \rightarrow 1$.

End of the proof of Theorem 1. Fix $s_{0}$ so that $a=a\left(s_{0}\right) \neq 0$. We need to find a lower bound for $s_{0}$. To this end we first define an increasing sequence $s_{0}, s_{1}, \ldots, s_{N}$ by

$$
s_{j}:=\sup \left\{s: a(s) \leq \lim _{t \rightarrow s_{j-1}+} e a(t)\right\} .
$$

Then

$$
\lim _{t \rightarrow s_{j}-} a(t) \leq \lim _{t \rightarrow s_{j-1}+} e a(t) \quad \text { and } \quad a\left(s_{j}\right) \geq e a\left(s_{j-2}\right) .
$$

We continue this process till

$$
1 \leq a\left(s_{N}\right)
$$

For fixed $s$ and $s^{\prime}$ such that $a(s) \leq e a\left(s^{\prime}\right)$ and $t:=s-s^{\prime}$ we have by the above two propositions

$$
\begin{aligned}
a\left(s^{\prime}\right) & \leq t^{-n} b(s) \leq A t^{-n} a(s) h^{-1}\left([a(s)]^{-1 / n}\right) \\
& =\operatorname{Aet}^{-n} a\left(s^{\prime}\right) h^{-1}\left([a(s)]^{-1 / n}\right) .
\end{aligned}
$$

Hence

$$
t \leq(A e)^{1 / n} h_{1}(a(s))
$$

where $h_{1}(x):=h^{-1 / n}\left(x^{-1 / n}\right)$. Letting $s \rightarrow s_{j+1}-$ and $s^{\prime} \rightarrow s_{j}+$ we thus get

$$
t_{j}:=s_{j+1}-s_{j} \leq(A e)^{1 / n} h_{1}\left(a\left(s_{j+1}\right)\right) .
$$

Since the function $h_{2}(x):=h_{1}\left(e^{x}\right)=h^{-1 / n}\left(e^{-x / n}\right)$ is increasing we can further estimate 
$(2)$

$$
\begin{aligned}
\sum_{j=0}^{N-1} t_{j} & \leq(A e)^{1 / n} \sum_{j=0}^{N-1} h_{2}\left(\log a\left(s_{j+1}\right)\right) \\
& \leq(A e)^{1 / n}\left(\sum_{j=0}^{N-2} \int_{\log a\left(s_{j}\right)}^{\log a\left(s_{j+2}\right)} h_{2}(x) d x+2 h_{2}\left(\log a\left(s_{N}\right)\right)\right) \\
& \leq 2(A e)^{1 / n}\left(\int_{-\infty}^{0} h_{2}(x) d x+h_{2}(\infty)\right)
\end{aligned}
$$

By our hypothesis on $h$, we have $h_{2}(\infty) \leq 1$ and

$$
\begin{aligned}
\int_{-\infty}^{0} h_{2}(x) d x & =\int_{-\infty}^{0} h^{-1 / n}\left(e^{-x / n}\right) d x \\
& =n \int_{1}^{\infty} h^{-1 / n}(y) y^{-1} d y=: n c(h)<\infty .
\end{aligned}
$$

These remarks combined with (2) give

$$
s_{N}-s_{0}=\sum_{j=0}^{N-1} t_{j} \leq 2(A e)^{1 / n}(n c(h)+1)=: c .
$$

This means that for $s^{\prime} \geq s_{0}+c$ we have $a\left(s^{\prime}\right)>1$ (see (1)). So fixing $s^{\prime}=s_{0}+c+1$ we conclude that $s^{\prime} \geq 0$ because otherwise, by applying Proposition 2, we would get a contradiction with the assumptions:

$$
\mu\left(U_{s^{\prime}}\right)>1 \text {. }
$$

Thus $s_{0} \geq-c-1=: B$. The proof is complete.

Remark. The hypothesis that $\mu$ satisfies $(* *)$ can be replaced by

$$
\mu(K) \leq A \operatorname{cap}(K) h^{-1}\left((\operatorname{cap}(K))^{-1 / n}\right)
$$

for any $K \subset \Omega$ compact and regular. The above proof still works.

It turns out that the integral condition $(* *)$ is not far from being sharp. From [BL, Corollary 2.2] (see also [D, Th. 2.2]) it follows that any bounded solution of $(*)$ satisfies $(* *)$ with $h \equiv 1$ and $A=n !\|u\|_{L^{\infty}}^{n} \int_{\Omega} d \mu$. However, if we let $h \equiv 1$ then $(* *)$ ceases to be a sufficient condition for boundedness of $u$ (when $n>1$ ). This can be seen by considering radial psh functions in a ball $B=B(0, R)$. In that case we have a characterization of bounded solutions of $(*)$ given in $[\mathrm{P}]$ (see also $[\mathrm{M}]$ ). A radial psh function $u$ is bounded if and only if

$$
\int_{0}^{R} r^{-1} F^{1 / n}(r) d r<\infty,
$$

where $F(r)=\int_{B(0, r)}\left(d d^{c} u\right)^{n}$. 
It is easy to see that for the rotation invariant measure $d \mu=\left(d d^{c} u\right)^{n}$ the integral in (**) assumes its maximal value for $v(z)=(2 \pi)^{-n} \log |z|$. Suppose that

$$
(2 \pi)^{n} \int_{B}|v|^{n} d \mu=\int_{0}^{R}|\log r|^{n} F^{\prime}(r) d r<\infty .
$$

Via integration by parts this is equivalent to

$$
\int_{0}^{R}|\log r|^{n-1} r^{-1} F(r) d r<\infty .
$$

Write $F(r)=|\log r|^{-n} g^{-1}(r)$. Then (4) takes the form

$$
\int_{0}^{R}[|\log r| r g(r)]^{-1} d r<\infty,
$$

whereas (3) now says

$$
\int_{0}^{R}\left[|\log r| r g^{1 / n}(r)\right]^{-1} d r<\infty .
$$

Taking $g$ such that the former inequality is satisfied but the latter is not, e.g. $g(r)=(\log |\log (r)|)^{n}$, we arrive at the desired conclusion.

Coupling Theorem 1 above with Theorem 1 from [KO] we obtain a fairly general class of measures for which the Dirichlet problem $(*)$ is solvable. For the definition of a measure locally dominated by capacity which we need in the statement of the next theorem we refer to [KO]. Essentially we require from such a measure (say $\mu$ ) that there exists $c>0$ such that given two concentric balls $B_{1}:=B(a, r) \subset B_{2}:=B(a, 2 r) \subset \Omega$ and a compact subset $E \subset B_{1}$, the following estimate holds:

$$
\mu(E) \leq c \mu\left(B_{1}\right) \operatorname{cap}\left(E, B_{2}\right) .
$$

(The actual definition is a bit less restrictive.)

THEOREM 2. If a measure $\mu$ in $\Omega$ is locally dominated by capacity and satisfies the condition (**) from Theorem 1 with h such that

$$
h(a x) \leq b h(x), \quad x>0,
$$

for some $a>1$ and $b>1$, then there exists a solution of $(*)$.

P r o o f. For a while we assume that $\mu$ has compact support in $\Omega$. Define a regularizing sequence of measures $\mu_{t}$ by fixing a radial non-negative function $\omega \in C_{0}^{\infty}(B)$ with $\int \omega d \lambda=1$ (here $B$ is the unit ball in $\mathbb{C}^{n}$ ) and setting

$$
\mu_{t}=\omega_{t} * \mu, \quad \text { where } \omega_{t}(z)=t^{-2 n} \omega(z / t), \quad t>0 .
$$


By Theorem 1 and Remark following it, it is enough to find $t_{0}>0$ and $A>0$ such that for any compact set $K \subset \Omega$,

$$
\mu_{t}(K) \leq A \operatorname{cap}(K, \Omega) h^{-1}\left((\operatorname{cap}(K, \Omega))^{-1 / n}\right), \quad t<t_{0} .
$$

Proposition 3. If $E \Subset \Omega$ is regular then for any $d>1$ there exists $t_{0}$ such that

$$
\operatorname{cap}\left(K_{y}, \Omega\right) \leq d \operatorname{cap}(K, \Omega), \quad|y|<t_{0},
$$

where $K \subset E$ is regular and $K_{y}:=\{x: x-y \in K\}$.

Proof. For $K \subset E$ define $w_{y}:=u_{K_{y}}(x+y)$, where $u_{K_{y}}$ is the extremal function of $K_{y}$. For any $c$ such that $0<c<1 / 2$ define $\Omega_{c}=\left\{u_{E}<-c\right\}$. By continuity of $u_{E}$ one can fix $t_{0}>0$ such that if $|y| \leq t_{0}$ and $x \in \Omega_{c / 2}$ then $x+y \in \Omega$. Therefore

$$
g(x):= \begin{cases}\max \left(w_{y}-c,(1+2 c) u_{E}\right)(x), & x \in \Omega_{c / 2}, \\ (1+2 c) u_{E}(x), & x \notin \Omega_{c / 2},\end{cases}
$$

is a well defined plurisubharmonic function in $\Omega$. Since $K \subset E$ and $w_{y}=-1$ on $K$ one concludes that $g=w_{y}-c$ in a neighbourhood of $K$. Hence

$$
\begin{aligned}
\operatorname{cap}(K, \Omega) & \geq(1+2 c)^{-n} \int_{K}\left(d d^{c} g\right)^{n}=(1+2 c)^{-n} \int_{K}\left(d d^{c} w_{y}\right)^{n} \\
& =(1+2 c)^{-n} \int_{K_{y}}\left(d d^{c} u_{K_{y}}\right)^{n}=(1+2 c)^{-n} \operatorname{cap}\left(K_{y}, \Omega\right) .
\end{aligned}
$$

Thus the proposition is proved.

To complete the proof of Theorem 2 let us fix a set $E$ and a positive number $t_{0}$ such that the above proposition holds with $E:=\bigcup_{t<t_{0}} \operatorname{supp} \mu_{t}$ $\Subset \Omega$ and $d=a^{n}$. By the assumptions there exists $A_{0}>0$ such that

$$
\mu(K) \leq A_{0} \operatorname{cap}(K) h^{-1}\left((\operatorname{cap}(K))^{-1 / n}\right) .
$$

Hence for $t<t_{0}$ we have by Proposition 3 and the extra assumption on $h$,

$$
\begin{aligned}
\mu_{t}(K) & \leq \sup _{|y|<t} \mu\left(K_{y}\right) \leq A_{0} \sup _{|y|<t} \operatorname{cap}\left(K_{y}\right) h^{-1}\left(\left(\operatorname{cap}\left(K_{y}\right)\right)^{-1 / n}\right) \\
& \leq A_{0} d \operatorname{cap}(K) h^{-1}\left((d \operatorname{cap}(K))^{-1 / n}\right) \\
& \leq A_{0} d b^{1 / n} \operatorname{cap}(K) h^{-1}\left((\operatorname{cap}(K))^{-1 / n}\right) .
\end{aligned}
$$

Setting $A:=A_{0} a^{n} b^{1 / n}$ we verify this way that $\mu_{t}$ satisfies $(\iota)$ for $t<t_{0}$, with the constant $A$ independent of $t$. Thus by Theorem 1 the family of solutions of $(*)$ for $\mu_{t}, t<t_{0}$, is uniformly bounded. So one can apply $[\mathrm{KO}$, Th. 1] to get the conclusion.

To verify the statement for an arbitrary measure $\mu$ note that by the above argument the solutions exist for $\chi_{j} d \mu$, where $\chi_{j}$ is a non-decreasing sequence of smooth cut-off functions with $\chi_{j} \uparrow 1$ in $\Omega$. Moreover, the $L^{\infty}$ 
norms of those solutions are uniformly bounded by a constant depending only on $A$. Hence the result follows by applying the monotone convergence theorem of [BT2].

Solutions for measures having densities in $L^{p}, p>1$. In Theorem 3 we are going to prove that for $d \mu=f d \lambda, f \in L^{p}(\Omega), p>1$, the Dirichlet problem $(*)$ has a continuous solution. To this end we shall use the following

Lemma 1. Suppose $v \in \operatorname{PSH}(\Omega) \cap C(\bar{\Omega}), v=0$ on $\partial \Omega$ and $\int\left(d d^{c} v\right)^{n}=1$. Then the Lebesgue measure $\lambda\left(U_{s}\right)$ of the set $\{v<s\}$ is bounded from above by $c \exp (-2 \pi|s|)$, where $c$ does not depend on $v$.

Proof. The proof is a variation of the proof of Proposition 2 of $[\mathrm{KO}]$. First we shall estimate $\operatorname{cap}\left(U_{s}\right)=\operatorname{cap}\left(U_{s}, \Omega\right)$ applying the Comparison Principle [BT2]. For $t>1$ and a regular compact set $K \subset U_{s}$ we have

$$
\operatorname{cap}(K)=\int_{K}\left(d d^{c} u_{K}\right)^{n}=\int_{\left\{-t s^{-1} v<u_{K}\right\}}\left(d d^{c} u_{K}\right)^{n} \leq t^{n} s^{-n} \int_{\Omega}\left(d d^{c} v\right)^{n} \leq t^{n} s^{-n} .
$$

Hence

$$
\operatorname{cap}\left(U_{s}\right) \leq|s|^{-n} .
$$

Write $\left(z_{1}, z^{\prime}\right) \in \mathbb{C} \times \mathbb{C}^{n-1}$ and set $U_{s}\left(z^{\prime}\right):=\left\{z_{1} \in \mathbb{C}:\left(z_{1}, z^{\prime}\right) \in U_{s}\right\}$. Let $V_{z^{\prime}}$ (resp. $V$ ) be the extremal function of logarithmic growth of $U_{s}\left(z^{\prime}\right)$ (resp. $\left.U_{s}\right)$. Then (see $\left.[\mathrm{TS}]\right)$

$$
\lambda\left(U_{s}\left(z^{\prime}\right)\right) \leq C_{1} T_{R}\left(U_{s}\left(z^{\prime}\right)\right),
$$

where $\lambda$ denotes the Lebesgue measure in $\mathbb{C}, C_{1}$ is an independent constant and

$$
T_{R}\left(U_{s}\left(z^{\prime}\right)\right):=\exp \left(-\sup _{\left|z_{1}\right|<R} V_{z^{\prime}}\right)
$$

with $R$ chosen so that $\Omega \subset B(0, R)$. Thus

$$
\begin{aligned}
\lambda\left(U_{s}\right) & =\int \lambda\left(U_{s}\left(z^{\prime}\right)\right) d \lambda\left(z^{\prime}\right) \leq C_{1} \int T_{R}\left(U_{s}\left(z^{\prime}\right)\right) d \lambda\left(z^{\prime}\right) \\
& =C_{1} \int \exp \left(-\sup _{\left|z_{1}\right|<R} V\left(z_{1}, z^{\prime}\right)\right) d \lambda\left(z^{\prime}\right) .
\end{aligned}
$$

A simple argument using a result of Alexander $[\mathrm{A}]$ shows that the right hand side of (6) is dominated by

$$
C_{2} \exp \left(-\sup _{|z|<R} V(z)\right)=C_{2} T_{R}\left(U_{s}\right)
$$

(see $[\mathrm{KO}]$ for details). Finally, we apply an inequality between the capacities cap and $T$ proved in $[\mathrm{AT}]$ to obtain

$\lambda\left(U_{s}\right) \leq C_{2} \exp \left[-2 \pi\left(\operatorname{cap}\left(U_{s}, B(0, R)\right)\right)^{-1 / n}\right] \leq C_{2} \exp \left[-2 \pi\left(\operatorname{cap}\left(U_{s}, \Omega\right)\right)^{-1 / n}\right]$. 
Hence by (5) we get

$$
\lambda\left(U_{s}\right) \leq C_{2} \exp (-2 \pi|s|)
$$

which was to be proved.

Corollary. If $v \in \operatorname{PSH}(\Omega) \cap C(\bar{\Omega}), v=0$ on $\partial \Omega$ and $\int_{\Omega}\left(d d^{c} v\right)^{n} \leq 1$, then $\|v\|_{L^{p}} \leq c(p)$.

Proof. By the lemma,

$\int|v|^{p} d \lambda \leq \int_{\Omega} d \lambda+\sum_{s=1}^{\infty} \int_{\{-s-1<v<-s\}}|v|^{p} d \lambda \leq c \sum_{s=1}^{\infty}(s+1)^{p} e^{-2 \pi s}=: c(p)<\infty$.

Now we are in a position to prove

Theorem 3. If $f \in L^{p}(\Omega, d \lambda), p>1, f \geq 0$ then the Dirichlet problem $(*)$ has a continuous solution for $d \mu=f d \lambda$.

$\operatorname{Proof}$. Set $f_{j}:=\min (f, j)$. Let $u_{j}$ be the continuous solution of

$$
\begin{aligned}
& \left(d d^{c} u\right)^{n}=f_{j} d \lambda, \\
& \lim _{z^{\prime} \rightarrow z} u\left(z^{\prime}\right)=\phi(z), \quad z \in \partial \Omega
\end{aligned}
$$

(see $[\mathrm{C}],[\mathrm{CP}]$ ). Then by the convergence theorem of [BT2], $u=\lim u_{j}$ is the desired solution provided $u_{j}$ is uniformly bounded. This is the case if the integral condition $(* *)$ in Theorem 1 is satisfied for $d \mu=f d \lambda$ and some suitable $h$. Let us verify this condition for $h(x)=\max (1, x)$. By Hölder's inequality we have

$$
\int|v|^{n} h(|v|) f d \lambda=\int_{\{v \geq-1\}}+\int_{\{v<-1\}} \leq\|f\|_{L^{1}}+\left(\int|v|^{(n+1) q} d \lambda\right)^{1 / q}\|f\|_{L^{p}}
$$

where $p^{-1}+q^{-1}=1$. Since by the Corollary above,

$$
\int|v|^{(n+1) q} d \lambda \leq c(q(n+1)),
$$

one can apply Theorem 1 to conclude that $u=\lim u_{j}$ is bounded.

Now, if $u_{j k}$ solves $\left(d d^{c} u\right)^{n}=\left|f_{j}-f_{k}\right| d \lambda, u=0$ on $\partial \Omega$, then by the Comparison Principle and the above argument,

$$
\left\|u_{j}-u_{k}\right\| \leq-u_{j k} \leq c_{p}\left\|f_{j}-f_{k}\right\|_{L^{p}}^{1 / n} .
$$

So $u_{j}$ is uniformly convergent and $u$ is continuous.

The last result readily extends to cover densities belonging to some Orlicz spaces. As an example (which can be refined yet) we give the following

Theorem 4. Let $L^{\varphi}(\Omega, d \lambda)$ denote the Orlicz space corresponding to $\varphi(t)=|t|(\log (1+|t|))^{n} h(\log (1+|t|))$ with $h$ satisfying the hypothesis of Theorem 1. If $f \in L^{\varphi}(\Omega, d \lambda)$ then $(*)$ is solvable with $d \mu=f d \lambda$. 
Proof. As in the preceding proof, it is enough to verify the condition $(* *)$. We apply Young's inequality for the function $g(\log (1+r))=$ $(\log (1+r))^{n} h(\log (1+r))$ and its inverse. Then

$$
\begin{aligned}
g(|v(x)|) f(x) & \leq \int_{0}^{f(x)} g(\log (1+r)) d r+\int_{0}^{g(|v(x)|)}\left[\exp \left(g^{-1}(t)\right)-1\right] d t \\
& \leq f(x) g(\log (1+f(x)))+\int_{0}^{|v(x)|} e^{s} g^{\prime}(s) d s \\
& \leq\|f\|_{L^{\varphi}}+g(|v(x)|) e^{|v(x)|} .
\end{aligned}
$$

When integrated over $\Omega$, the right hand side remains bounded since by the lemma,

$$
\int_{\Omega} g(|v(x)|) e^{|v(x)|} d x \leq c \sum_{s=1}^{\infty} e^{s(1-2 \pi)} g(s+1)<\infty .
$$

Thus the result follows from Theorem 1 .

EXAMPle. If $\varphi(t)=|t|(\log (1+|t|))^{n}(\log (\log (1+|t|)))^{m}, m>n$, then Theorem 4 applies. On the other hand, if $\varphi(t)=|t|(\log (1+|t|))^{m}, m<n$, it is no longer true; a suitable counterexample is given in $[\mathrm{P}]$.

\section{References}

[A] H. Alexander, Projective capacity, in: Conference on Several Complex Variables, Ann. of Math. Stud. 100, Princeton Univ. Press, 1981, 3-27.

[AT] H. Alexander and B. A. Taylor, Comparison of two capacities in $\mathbb{C}^{n}$, Math. Z. 186 (1984), 407-417.

[B] E. Bedford, Survey of pluri-potential theory, in: Several Complex Variables: Proceedings of the Mittag-Leffler Inst. 1987-1988, J. E. Fornaess (ed.), Math. Notes 38, Princeton University Press, 1993, 48-97.

[BT1] E. Bedford and B. A. Taylor, The Dirichlet problem for a complex MongeAmpère operator, Invent. Math. 37 (1976), 1-44.

[BT2] -, 一, A new capacity for plurisubharmonic functions, Acta Math. 149 (1982), $1-40$.

[BL] Z. Błocki, Estimates for the complex Monge-Ampère operator, Bull. Polish Acad. Sci. Math. 41 (1993), 151-157.

[C] U. Cegrell, On the Dirichlet problem for the complex Monge-Ampère operator, Math. Z. 185 (1984), 247-251.

[CP] U. Cegrell and L. Persson, The Dirichlet problem for the complex MongeAmpère operator: stability in $L^{2}$, Michigan Math. J. 39 (1992), 145-151.

[CS] U. Cegrell and A. Sadullaev, Approximation of plurisubharmonic functions and the Dirichlet problem for the complex Monge-Ampère operator, Math. Scand. 71 (1993), 62-68.

[D] J.-P. Demailly, Mesures de Monge-Ampère et caractérisation géométrique des variétés algébriques affines, Mém. Soc. Math. France (N.S.) 19 (1985). 
[K] M. Klimek, Pluripotential Theory, Oxford University Press, 1991.

[KO] S. Kołodziej, The range of the complex Monge-Ampère operator, Indiana Univ. Math. J. 43 (1994), 1321-1338.

[M] D. R. Monn, Regularity of the complex Monge-Ampère equation for radially symmetric functions of the unit ball, Math. Ann. 275 (1986), 501-511.

[P] L. Persson, On the Dirichlet problem for the complex Monge-Ampère operator, Doctoral Thesis No 1, 1992, University of Umeå.

[S] J. Siciak, Extremal Plurisubharmonic Functions and Capacities in $\mathbb{C}^{n}$, Sophia University, Tokyo, 1982.

[TS] M. Tsuji, Potential Theory in Modern Function Theory, Tokyo, 1959.

Institute of Mathematics

Jagiellonian University

Reymonta 4

30-059 Kraków, Poland

E-mail: kolodzie@im.uj.edu.pl

Reçu par la Rédaction le 10.9.1994

Révisé le 20.1.1995 